\title{
Multi-spectral study of a new sample of blue compact dwarf galaxies
}

\section{I. $B$ and $R$ surface photometry of 23 objects from the Byurakan lists ${ }^{\star}$}

\author{
V. Doublier ${ }^{1,4}$, G. Comte ${ }^{1}$, A. Petrosian ${ }^{2}$, C. Surace ${ }^{1}$, and M. Turatto ${ }^{3}$ \\ 1 Observatoire de Marseille and Institut Gassendi, (CNRS), 2 place Le Verrier, F-13248 Marseille Cedex 04, France \\ 2 Byurakan Astrophysical Observatory, Ashtarak region, 378433 Armenia \\ 3 Osservatorio Astronomico di Padova, 5 Vicolo dell'Osservatorio, I-35122 Padova, Italy \\ 4 European Southern Observatory, Karl-Schwarzschild-Strasse 2, D-8046 Garching bei München, Germany
}

Received March 15; accepted November 27, 1996

\begin{abstract}
We present the first results of the surface photometry of a sample of Blue Compact Dwarf galaxies (hereafter BCDGs). The images were obtained at Pic du Midi (France) and Asiago (Italy). In this paper, we produce an atlas of isophotal maps and brightness distribution profiles for 23 objects in the Northern hemisphere. Short individual descriptions of the galaxies, and tables of photometric parameters are given.

The main result is that a substantial fraction of objects are showing an $r^{1 / 4}$ brightness distribution, consistently with previous observations. The integral colors, luminosity-radius relations and "compactness" properties are briefly investigated. This sample is being extended with new observations, that will be reported in a future paper with a more complete discussion.
\end{abstract}

Key words: galaxies: compact - galaxies: photometry - galaxies: starburst — galaxies: structure

\section{Introduction}

Numerous studies performed on dwarf galaxies have shown large differences in observable properties as the morphology, color and stellar content. Beside the wellknown classes of dwarf spheroidal/dwarf elliptical systems and dwarf Magellanic (disky) systems the so-called Blue Compact Dwarf Galaxies (hereafter BCDGs), originally defined by Zwicky $(1967,1971)$ and, later discussed by

Send offprint requests to: V. Doublier

* Based on observations performed at Bernard Lyot Telescope, Pic-du-Midi, operated by Institut National des Sciences de l'Univers, CNRS, and at Cima Ekar telescope, operated by Padova Observatory.
Thuan \& Martin (1981), have focused attention after the discovery by Sargent \& Searle (1970) that some of them were low metallicity systems. BCDGs show metallicity ranging from $1 / 6$ to $1 / 50$ solar value (extreme case being I Zw 18: Searle \& Sargent 1972; Davidson \& Kinman 1985; Kunth \& Sargent 1986). These systems are known to host a very active stellar formation. Scaled to the low total luminosity of these dwarf objects with absolute magnitudes $M_{b} \geq-16$, this corresponds to star formation rates able to exhaust the available gas content of the galaxy in a time much smaller than the age of the Universe.

It is quite surprising however that only few quantitative imaging studies of the brightness distribution in BCDGs have been published insofar. Barbieri et al. (1979) performed a pioneering investigation of 15 objects on photographic plate material, suggesting that some of them could be considered as dwarf elliptical systems. Loose \& Thuan (1983) have reported preliminary qualitative results on a sample of 22 BCDGs, separating 4 morphological classes based on the shape of the isophotes and the apparent distribution of the star forming knots. Loose \& Thuan (1985) have also made a detailed study of Haro 2, a moderate luminosity spheroidal object housing a strong star formation activity in its center. Kunth et al. (1988), from a multicolor deep CCD imagery of a restricted sample of 7 objects, have shown that some BCDGs obey a quasi-pure $r^{1 / 4}$ law, as do giant ellipticals, while others are assumed to be disk-like systems, since they obey an exponential law. Recently, Papaderos et al. (1996) discussed such morphological properties, using profile decomposition, for some bright BCDGs. Some objects (e.g. Pox 186) have so small apparent diameters that no firm conclusions could be derived. BCDGs thus form "a mixed bag of morphological types", probably reflecting widely different evolutionary histories. The blue-excess starburst region is in some cases surrounded by a red envelope suggesting the 
presence of an evolved red giant population (Aaronson 1986; Thuan 1983). Strong color gradients might indicate, beside a change in the metallicity, the existence of old stars of population II. Other authors (Bothun et al. 1989; Salzer et al. 1991) have published images obtained in follow-up studies of active galaxies, but only a few BCDGs are studied in these papers. The case of IZw18 has attracted much more attention because of its extreme abundance characteristics and deep detailed imagery in continuum and emission lines has been published by Hua et al. (1987), Davidson et al. (1989), Dufour \& Hester (1990) and Petrosian et al. (1996) while recently Hubble Space Telescope imaging allowed Hunter \& Thornson (1995) to build the color-magnitude diagram of the massive star population.

Our purpose is to investigate the brightness distributions of BCDGs, their structure (presence of one or several areas of star formation, of disks, spheroids, of nonaxisymmetric components, traces of possible interactions, color gradients etc...) and to search for relationships, if any exist, between these parameters and the spectroscopic indicators of galactic evolution such as the metallicity of the ionized gas or the estimation of the age of the starburst. Hence, the imaging and photometric observing program whose results are reported here is coupled with a program of detailed, high spatial resolution spectro-imaging observations that will be reported elsewhere.

\section{The sample}

In the following, we describe the results obtained on a sample of BCDGs selected from the two Surveys conducted at Byurakan Observatory:

- the First Byurakan Survey (FBS) or Markaryan survey (Markaryan 1967 and following papers), on which thoroughly complete information is given by Mazzarella \& Balzano (1986),

- the Second Byurakan Survey (SBS) (Markaryan \& Stepanian 1983, and following papers) which spans a restricted area in the Northern Hemisphere but is 2 magnitude deeper than the FBS in the average.

Both surveys use a color criterion (the ultraviolet excess in the continuum) to select active galaxies. Most lowredshift, moderate or faint luminosity objects present in the FBS and the SBS exhibit an HII-like, narrow emission line spectrum. The presence of emission lines is a direct indicator of formation of massive ionizing stars, while the continuum ultraviolet excess is the signature of an integrated population containing a rich main-sequence of intermediate mass stars of $\mathrm{B}-\mathrm{A}-\mathrm{F}$ spectral types.

Our main interests lying in low-luminosity objects, we have selected BCDGs of low redshifts (typically lower than a recession velocity of $2000 \mathrm{~km} \mathrm{~s}^{-1}$ ) with estimates of absolute magnitude $M_{b} \geq-16.5$. At the distances implied by these small recession velocities, we could keep a sufficient image size even on small linear diameter objects and retrieve physically significant data from the surface photometry even with 1 arcsec seeing conditions. All the selected objects have been previously observed spectroscopically at low spectral resolution (Stepanian, private communication for SBS objects, and numerous other sources, see Mazzarella \& Balzano 1986) and have an HII-like emission line spectrum. Finally, objects with small angular size for the given redshift (typically 10 to $50^{\prime \prime}$ ) have been preferred in order to bias deliberately the sample towards dwarf "compact" galaxies.

Table 2 lists the observed objects and is selfexplanatory. The apparent magnitudes are photographic estimates from the Byurakan surveys. Note that some objects present in Table 2 are still unpublished (Stepanian, private communication).

\section{Observations and reduction}

\subsection{Observational material}

Most images were obtained at the Bernard Lyot $2 \mathrm{~m}$ telescope at Pic du Midi Observatory (France) using ISARD imaging all-reflective camera (Lemaitre et al. 1996, in preparation) using a $1060 \times 1024$ thick Thomson THX CCD with $19 \times 19 \mu$ pixels. ISARD gives a scale of 0.248 arcsec per pixel but in the seeing conditions we met this leads to some oversampling at the expense of exposure time. Therefore, the readout of the CCD was systematically done using a $2 \times 2$ pixels on-chip binning mode. Seeing conditions were between $2^{\prime \prime}$ (at worst) in November 92 and an exceptional 0.5" during a night of February 1994, but the average was around $1.2^{\prime \prime}$. For objects observed during different runs, we selected the frames taken under the best sky absorption/seeing conditions.

Several objects were observed at the $182 \mathrm{~cm}$ Cima Ekar telescope at Asiago Observatory (Italy), using a TK 512 CCD (with $0.3^{\prime \prime} / \mathrm{pix}$ ) during several runs from August 92 to March 94 . The average seeing conditions were around $2^{\prime \prime}$.

We used Johnson $B$ and Gunn $R$ filters. Coupled with the thick Thomson CCD quantum efficiency spectral distribution, we found that the $R$ instrumental band was easy to reduce to the standard Cousins $R$ band with very small color term in the reduction equation. The color term was a little bit larger for the reduction of the $B$ instrumental band to the standard Johnson $B$ band. To derive the reduction equations, Landolt $(1983,1992)$ equatorial standard stars were observed, as well as, each night, M 67 "dipper asterism" field. (Schild 1983). The M 67 field was observed at two or three zenith distances to derive the extinction correction. The globular cluster NGC 7790 was also observed in December 1992. High photometric quality nights in February 94 at Pic du Midi allowed to search for the color extinction coefficient, which was found to be not significant $\left(k_{B-R}^{\prime} \sim 0.005\right)$ and therefore not taken into 
Table 1. List of selected objects

\begin{tabular}{|c|c|c|c|c|c|c|}
\hline Object name & $\alpha(2000)$ & $\delta(2000)$ & $z$ & mag. app. & Obs. period/ Telescope & photom. cond. \\
\hline$\overline{\text { SBS } 0136+328}$ & 013621.4 & 325150 & 0.0211 & 18. & Asiago Nov. $92^{*}$ & fair \\
\hline SBS $0940+544 \mathrm{C}$ & 094050.9 & 542520 & 0.0057 & 18. & TBL March $93^{* *}$ & good \\
\hline SBS $1006+578$ & 100611.9 & 574842 & 0.0045 & 17. & TBL Feb. $94 * *$ & v. good \\
\hline SBS $1054+504$ & 105402.5 & 502419 & 0.0046 & 16.5 & TBL Feb. $94 * *$ & v. good \\
\hline SBS $1147+520$ & 114716.4 & 520051 & 0.0036 & 16.5 & TBL March $93 * *$ & good \\
\hline SBS $1331+493$ & 133120.3 & 492135 & 0.0024 & 15. & Asiago March $94 *$ & fair \\
\hline SBS $1413+495$ & 141318.8 & 493424 & 0.0024 & 16. & Asiago March $94 *$ & fair \\
\hline SBS $1428+457$ & 142819.2 & 454554 & 0.0078 & 16. & TBL March $93^{* *}$ & good \\
\hline SBS $1533+574$ & 153303.5 & 572702 & 0.0107 & 17.5 & TBL March 93 ** & good \\
\hline MRK 324 & 232632 & 181558 & 0.0054 & 15.6 & Asiago Aug. $92 *$ & poor \\
\hline MRK 900 & 212959.5 & 022451 & 0.0038 & 14.3 & Asiago Aug. $92 *$ & poor \\
\hline MRK 996 & 012735.4 & -061936 & 0.0054 & 15.5 & Asiago Oct. $92 *$ & not photometric $(R)$ \\
\hline MRK 1131 & 232801.1 & -020953 & 0.0065 & 16. & Asiago Oct. $92 *$ & not photometric $(B$ and $R$ ) \\
\hline MRK 1308 & 115412.2 & 000811 & 0.0035 & 13.7 & TBL Feb. 94 ** & v. good \\
\hline MRK 1416 & 092055.9 & 523408 & 0.0072 & 17. & TBL March 93/Feb. $94 * *$ & $\operatorname{good} / \mathrm{v} \cdot \operatorname{good}$ \\
\hline MRK 1418 & 094027.2 & 482013 & 0.0015 & 13.5 & TBL Dec. $92 * *$ & good \\
\hline MRK 1423 & 094301.7 & 585824 & 0.0037 & 15.1 & TBL Dec. $92 * *$ & good \\
\hline MRK 1426 & 094918.4 & 483349 & 0.0062 & 16.5 & TBL March 93 ** & good \\
\hline MRK 1434 & 103410 & 580350 & 0.0068 & 16.5 & TBL March $93 * *$ & good \\
\hline MRK 1450 & 113835.6 & 575227 & 0.0029 & 16. & TBL March $93^{* *}$ & good \\
\hline MRK 1480 & 134251.7 & 524231 & 0.0058 & 16.5 & TBL March 93/Feb. $94 * *$ & $\operatorname{good} / \mathrm{v} \cdot \operatorname{good}$ \\
\hline MRK 1481 & 134259.3 & 524118 & 0.0060 & 17. & TBL Feb. $94 * *$ & v. good \\
\hline MRK 1499 & 163521.1 & 521253 & 0.0087 & 15.6 & TBL March $93^{* *}$ & good \\
\hline
\end{tabular}

$\left.{ }^{*}\right)$ : Asiago: $1.82 \mathrm{~m}$ Telescope, Cima Ekar.

$(* *)$ : TBL: Pic du Midi, 2 m Bernard Lyot Telescope.

account in the galaxy images reduction. Flat-fielding was done with frames taken on evening and morning twilight sky.

\subsection{Pre-reduction of the frames}

The data reduction was performed in 2 steps. First, the raw images were bias subtracted and flat-fielded. When a complete nightly set of flats was not available (because of observing conditions only favourable during part of the night) we used a procedure creating a mask of the images and we used as a flatfield the averaged and masked sky backgrounds. MIDAS package also provides a way of building a flatfield from the raw image. Both methods were used in order to avoid spurious effects like stars or faint objects in the field being taken into account during the process. The images from Asiago were treated along the same scheme.

In a second step the cosmic rays impacts were removed from the CCD frames. We largely used a standard MIDAS routine for this operation, checking very carefully that the information contained in the images were not altered, especially the centers of the stars and the maxima of brightness in the galaxies, which could be mistaken as cosmic rays impacts by the automated procedure in very sharp seeing conditions.

\subsection{Standard stars}

Careful aperture photometry was performed on the standard stars of the cluster fields and on the Landolt stars.
The following equations were used to reduce the instrumental magnitudes and colors to the Johnson-Cousins system:

$$
\begin{aligned}
& B=b_{\mathrm{TBL}}+(0.20)(b-r)_{\mathrm{TBL}}+24.55 \\
& R=r_{\mathrm{TBL}}+(0.075)(b-r)_{\mathrm{TBL}}+25.01
\end{aligned}
$$

at Bernard Lyot telescope

$$
\begin{aligned}
& B=b_{\mathrm{AS}}+(0.29)(b-r)_{\mathrm{AS}}+22.40 \\
& R=r_{\mathrm{AS}}+(0.12)(b-r)_{\mathrm{AS}}+23.00
\end{aligned}
$$

at Cima Ekar telescope.

where $b$ and $r$ are the instrumental magnitudes defined by:

$b=-2.5 \log \left(F_{b, \text { cor }}\right)$

$r=-2.5 \log \left(F_{r, \text { cor }}\right)$

$F_{b, \text { cor }}$ and $F_{r, \text { cor }}$ being the flux in ADU corrected from the sky background, per minute of exposure time.

The reference magnitudes for the M 67 stars were taken from Schild (1985), Gilliland et al. (1992) and Chevalier \& Ilovaisky (1991). Odewahn et al. (1992) provided the values for NGC 7790 stars.

The atmospheric absorption coefficients were determined on a nightly basis whenever possible through standard methods. These coefficients were then used to correct the magnitudes and surface brightnesses of the galaxies. 
The $R$ Gunn filter includes the $\mathrm{H} \alpha$ line in emission in the rest frame, and all objects having a low redshift, their $\mathrm{H} \alpha$ emission is always present in the measured $R$ flux. This effect is probably not dominant in the presence of a substantial background of evolved stellar population, but may contribute in some cases to produce local red excess inside the galaxies, especially on star-forming complexes that have a large equivalent width in emission. Note however that in the galaxy SBS $1331+493$, which contains a knot of high excitation and very large equivalent width in emission lines, the knot appears extremely blue in our photometry.

\subsection{Galaxy photometry}

\subsubsection{Data reduction}

The surface photometry of the galaxies was performed with specially designed MIDAS macro-procedures. Basically, we performed isophotal integration of the flux. First, the sky background is evaluated in a number of pixel sub-arrays surrounding the object. Further, the classical method of "equivalent profile" devised by de Vaucouleurs (1959) and applied by numerous other authors (see e.g. Ables 1971; Fraser 1977 for detailed explanations) has been used. The flux from the galaxy is computed inside isophotes of decreasing brightness $\mu$, without setting any constraint on the geometrical shape of the isophotes. This method allows to take into account irregular shapes occurring in star-forming regions, detached "islands" of brightness, etc... and is well adapted to non-axisymmetric objects. The brightness profile produced is called the "equivalent" profile. Each step of the isophotal integration produces the following information:

- "equivalent" radius: $r_{\mathrm{e}}$ is defined by:

$$
r_{\mathrm{e}}=\sqrt{A / \pi}
$$

where $A$ is the area of the isophote considered

- surface brightness at $r=r_{\mathrm{e}}$ :

$$
\mu_{\mathrm{e}}=-2.5 \log \left(I\left(r_{\mathrm{e}}\right)-I_{\mathrm{sky}}\right)
$$

where $I$ and $I_{\text {sky }}$ are the respective levels (in ADU per pixel per minute of exposure time) of the isophote and of the sky background. $\mu_{\mathrm{e}}$ is reduced to the $B, R$ standard system by application of the reduction Eqs. (1) to (4) after correction for atmospheric extinction. - integrated magnitude at $r=r_{\mathrm{e}}$ :

$m_{\mathrm{e}}=-2.5 \log \left(F-\left(I_{\text {sky }} \times A\right)\right)$

where $F$ is the total flux inside the isophote of area $A$ (expressed in pixels). $m_{\mathrm{e}}$ is reduced to the standard system after atmospheric extinction correction.

The sky background was checked for each frame to be sufficiently flat in the vicinity of the galaxies to avoid sky-background fitting and subtraction inducing complementary noise around the fainter isophotes. This was done using different sky fitting algorithms and computing the rms on the sky-background. The errors were estimated using the rms of the sky-background mean level around the galaxies. Typically, the errors were of less than a percent when no sky was subtracted and of about $1-5 \%$ after subtraction.

The quality of the isophotal integration critically depends on the local signal-to-noise ratio. The outermost parts of the galaxies are weak extended sources and their signal-to-noise ratio is generally insufficient on the direct images. To extend the photometry down to 1 percent of the sky background or even further, one must smooth the frames in some way. However, efficient smoothing numerical filters (such as the median filter) have generally poor local flux conservation properties, especially when strong brightness gradients are present in the image. Therefore, the isophotal integration was performed in two steps. For the inner parts of the galaxies, we used the direct full resolution frame, producing a first brightness profile. For the outer parts, we applied a gaussian filter of width slightly larger than the seeing disk before integrating and built a second brightness profile. The two brightness profiles were subsequently compared and merged together. This method proved satisfactory in all cases.

The data obtained at Asiago were also treated independently (by M.T.) using a method of ellipse fitting to derive the integrated magnitude and surface brightness profiles. This process fits ellipses to the isophotes without forcing the position of the ellipse centers. It gives results in excellent agreement with those produced by the equivalent de Vaucouleurs profile when applied to regular ellipticallike dwarfs. It fails on the central parts of the irregular-like dwarfs or the dwarf compacts showing several star formation regions surrounded by regular (elliptical or circular) shells.

The results of the isophotal integration are contained in two photometric tables $(B$ and $R$ ) for each object. From these tables, the following quantities are derived:

- asymptotic magnitudes and colors: the "asymptotic" (total) magnitude of a galaxy can be determined only through an extrapolation process, since the flux integration is always limited to some fraction of the sky background in excess of the latter. We used the following procedure: the integrated apparent magnitude was plotted versus $r_{\mathrm{e}}$ and an asymptotic value for this "growth curve" was searched interactively, with a simple parabolic extrapolation beyond the last three measured points.

- "effective" radius and brightness: these quantities were introduced by De Vaucouleurs to allow the comparison between various types of galaxies, and hence of brightness distributions, by means of "metric" data, that is, quantities that are independent of the brightness of the sky background. They are defined as: 
- $r_{\text {eff }}$ (effective equivalent radius): the equivalent radius of the isophote containing half the total luminosity of the object (i.e. the value of $r_{\mathrm{e}}$ for which the cumulated magnitude is equal to $B_{\text {asymp }}+0.75$ or $\left.R_{\text {asymp }}+0.75\right)$.

$-\mu_{\text {eff }}$ (effective surface brightness): the surface brightness at $r_{\mathrm{e}}=r_{\text {eff }}$

- the mean surface brightness inside the effective radius is also an interesting quantity related to the so-called "compactness" of the galaxy. It is derived from the effective apparent magnitude and the area of the effective isophote. The mean surface brightness inside the effective radius is given by:

$<\mu_{\text {eff }}>=m_{\text {eff }}+5 \log r_{\text {eff }}+2.5 \log \pi$

- a concentration index gives an idea of the relative importance of the inner parts of the object with respect to the outer parts as regard to the luminosity. It is also related to the "compactness" in the visual appearance of the image of the galaxy. The concentration index as defined by de Vaucouleurs (1959) is given by:

$C_{21}=\frac{r_{\mathrm{eff}}}{r_{0.25}}$

where $r_{0.25}$ is the radius at $1 / 4$ th of the total luminosity.

\subsubsection{Errors estimation}

The errors on the surface brightness profiles were estimated assuming that the Poisson statistics apply and taking into account the errors on the background subtraction (Saglia 1996).

For the errors on the integrated magnitude, the Poisson statistics do not entirely apply as one has to take into account the extrapolation process and the limitation due to the quality of the observations. The deeper the better, in order to reach the asymptotic magnitude without extrapolating.

\subsection{3. $B-R$ color profiles}

Ideally, The $B-R$ profiles are derived from the $B-R$ maps obtained from the $B$ and $R$ images of the galaxies. Due to the limited $\mathrm{S} / \mathrm{N}$ ratios obtained in the outer faint regions on our $R$ band images, in order to analyze the color distribution of our BCDGs, we have adopted a method that uses the SB profiles of the galaxy in $B$ and $R$ band. The principle is to subtract the two SB distributions to built the new $B-R$ distribution. We used the best sampled SB profile $(B$ or $R$ ) to interpolate the apparent surface brightnesses as function of the equivalent radius. This allows to sample the surface brightnesses following the same distribution of radius and to subtract them point by point. This method does not exactly reproduce the general variation in colors along the SB profiles when the isophotal integration centers are not the same in both bands, because the interpolated points do not correspond physicaly. However, the differences are smaller than the errors estimated on the color profiles.

The $B-R$ color distributions are shown in the atlas with the estimated errors (we assumed the errors in $B$ and $R$ to be independent, so the $\left.\sigma_{B-R}=\sqrt{\sigma_{B}^{2}+\sigma_{R}^{2}}\right)$.

\subsubsection{Results of the photometry}

The results of the photometry are presented in an atlas (Fig. 1) and a table. For each galaxy, the isophotal map in $B$ (with three exceptions for which only $R$ images are available) is given and the equivalent surface brightness distributions in $B$ and $R$ are plotted using a linear scale for the radius, as well as the $B-R$ profile at the top of the plot. Table 2 collects the observable parameters derived from the surface photometry:

Column 1: Markaryan number or SBS name.

Column 2: Asymptotic apparent magnitude in $B$ and asymptotic $B-R$ color.

Column 3: Surface brightness inside the effective radius in mag $\operatorname{arcsec}^{-2}$.

Column 4: Mean surface brightness inside the effective radius.

Column 5: Equivalent effective radius in arcsec.

Column 6: Equivalent radius at 1/4 of the total luminosity in arcsec.

Column 7: Isophotal equivalent radius at $\mu=$ $24 \mathrm{mag} \operatorname{arcsec}^{-2}$ in arcsec.

Column 8: Concentration index in $B$ and $R$ colors.

The magnitudes were corrected from the galactic extinction, using the $E(B-V)$ maps of Burstein \& Heiles (1982). The absorption coefficient are computed from the $E(B-V)$ values using the equation given by Savage \& Mathis (1979):

$\frac{A_{\lambda}}{E(B-V)}=2.204 \times \lambda^{-1}-0.9078$.

Figure 2 shows the comparison of the measured asymptotic $B$ magnitudes with the catalog photographic magnitudes given by Mazzarella \& Balzano (1986), and the magnitudes given by Stepanian (private communication) for SBS objects. The CCD magnitudes appear consistent with the eye estimated magnitudes from the catalogs across the range spanned by the selected objects. The dispersion in the diagram is also consistent with the general accepted accuracy of \pm 0.5 mag of the Markaryan catalog apparent magnitudes.

\subsection{Comments on individual objects}

A large fraction of the galaxies show several knots of star formation activity, e.g. SBS1533+57 which shows at least 

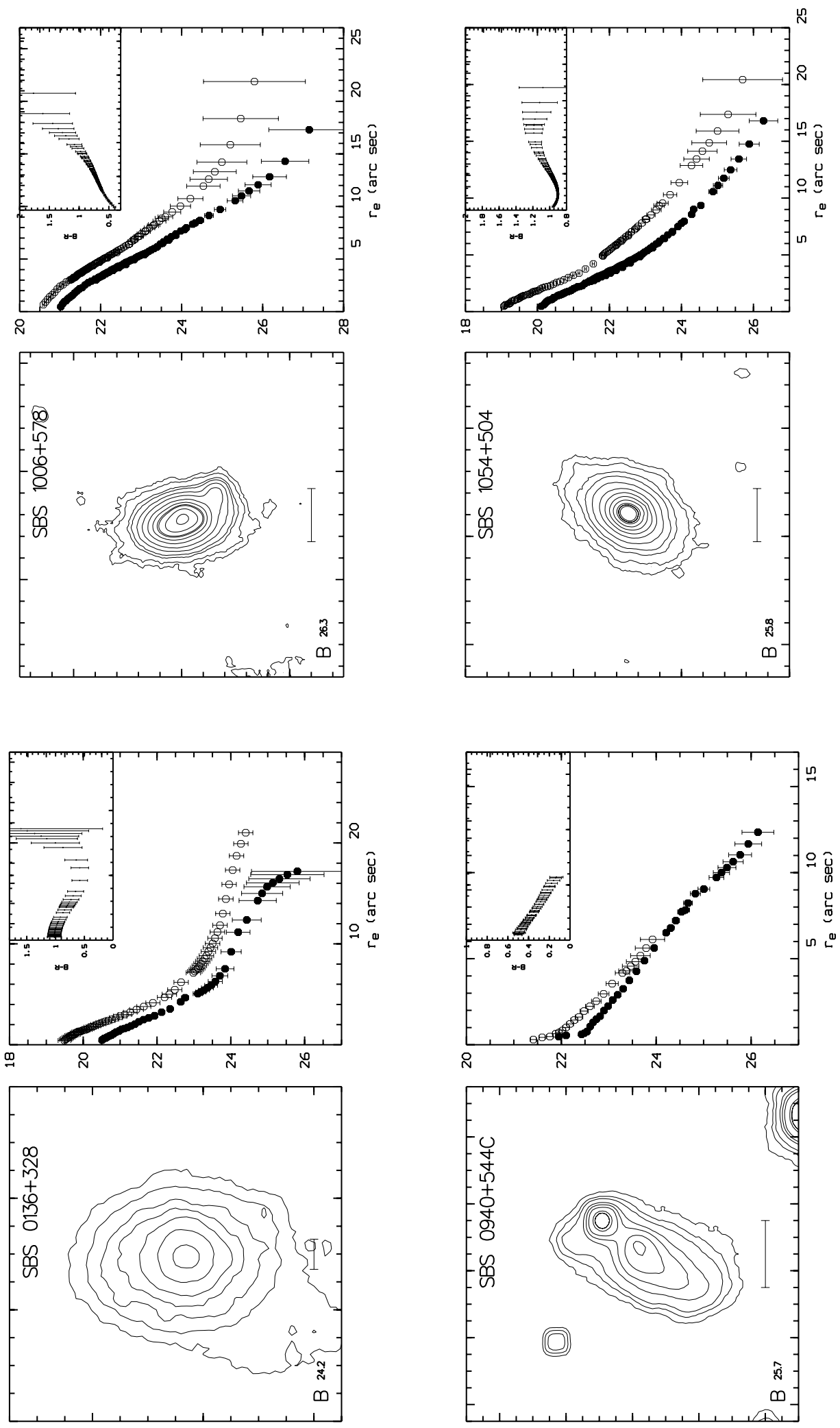

Fig. 1. Atlas of contour plots and photometric profiles. For each galaxy, the contour plot of the $B$ image is shown on the left panel, with a surface brightness interval of 0.5 magnitude per square arc second. The threshold surface brightness is indicated in the lower left corner. For Mk 1423, Mk 1426 and SBS $1533+574$, the $R$ maps are shown, no $B$ image being available. The solid horizontal bar has a length of $1 \mathrm{kpc}$. The right-hand panel displays the surface brightness distribution profiles (abscissa: equivalent radius in arc seconds, ordinate: surface brightness in magnitude per square arc second) in $B$ (dots) and $R$ (circles). (To be seen in landscape) 

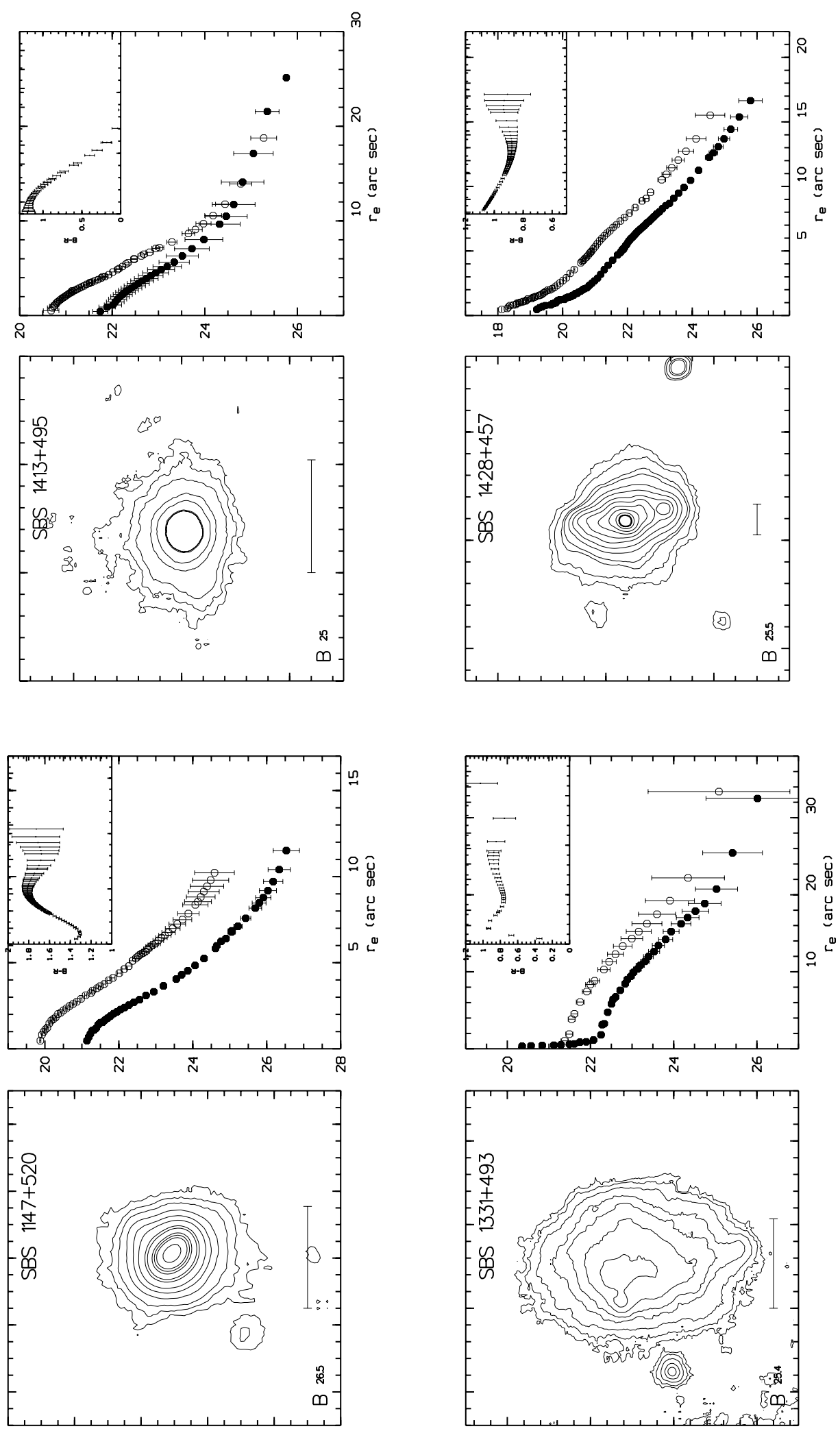

Fig. 1. continued. (To be seen in landscape) 

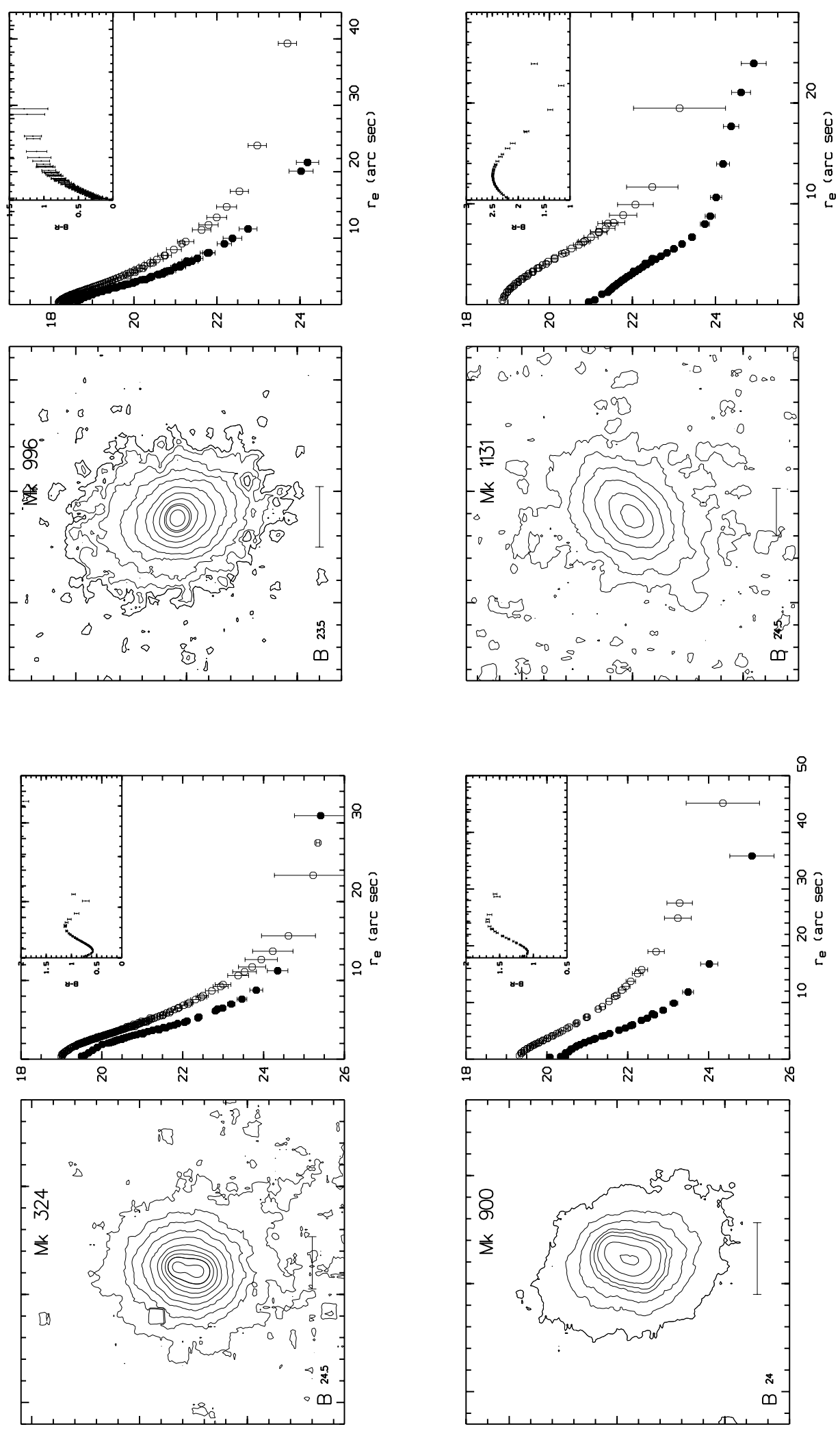

Fig. 1. continued. (To be seen in landscape) 

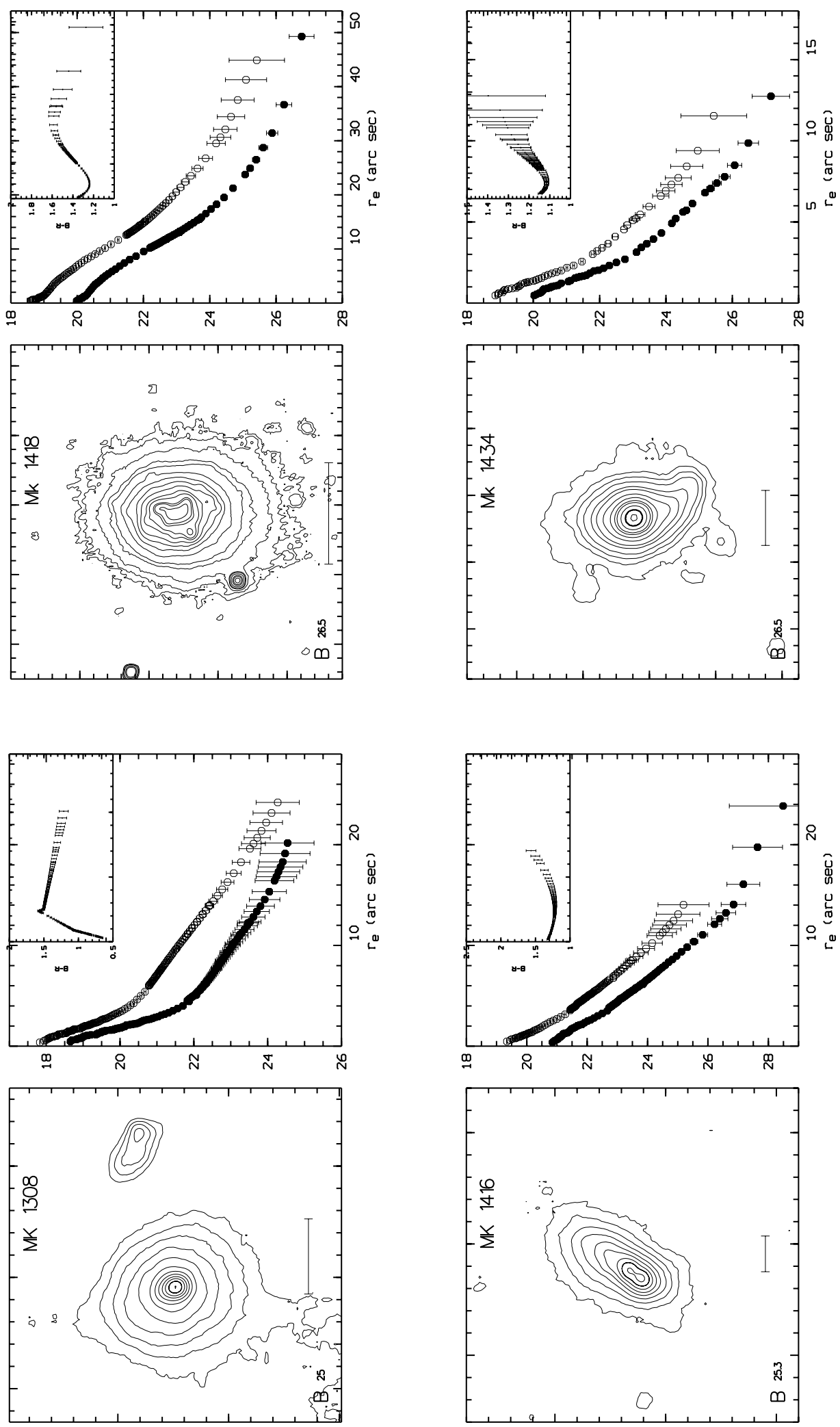

Fig. 1. continued. (To be seen in landscape) 

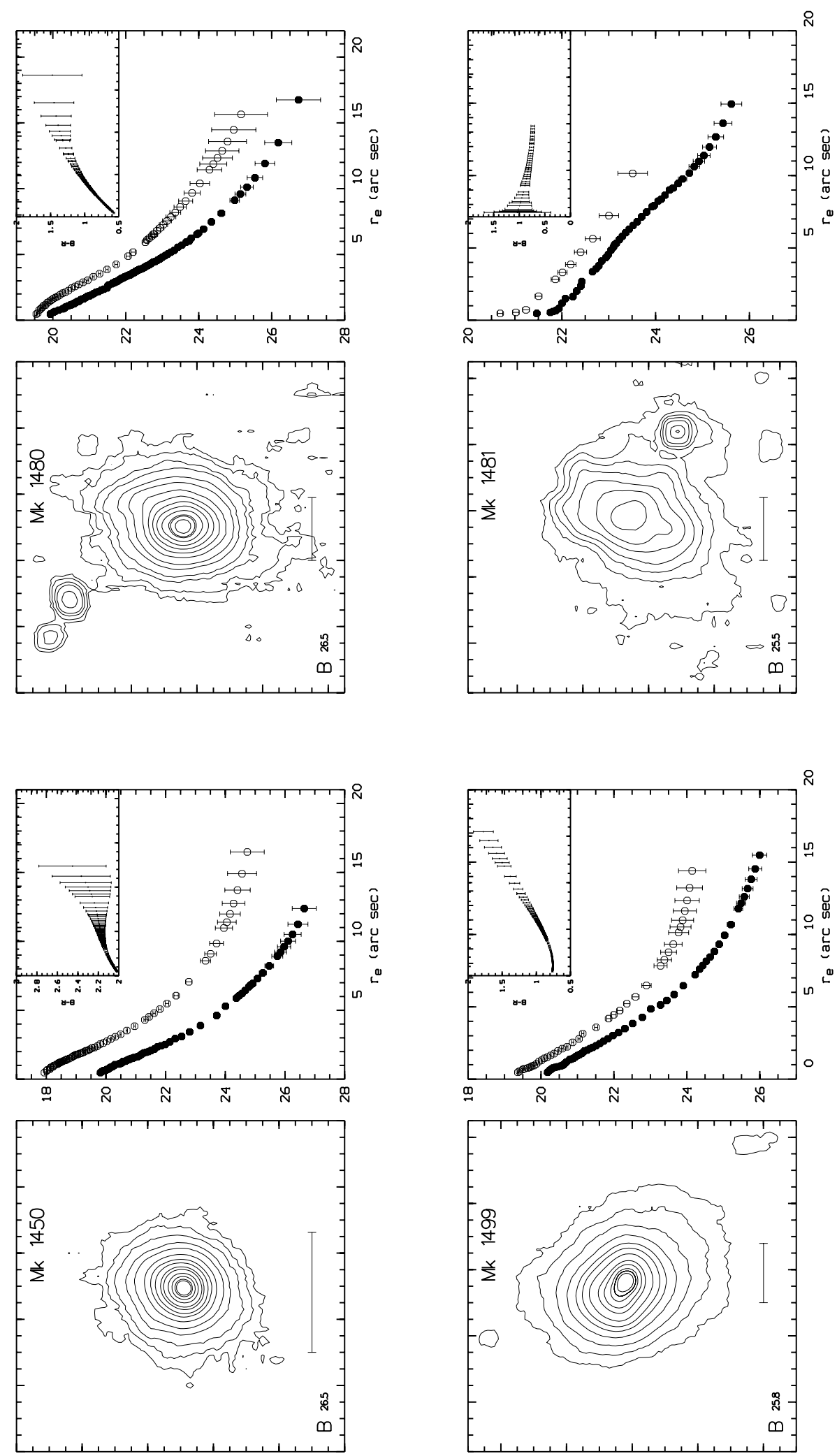

Fig. 1. continued. (To be seen in landscape) 

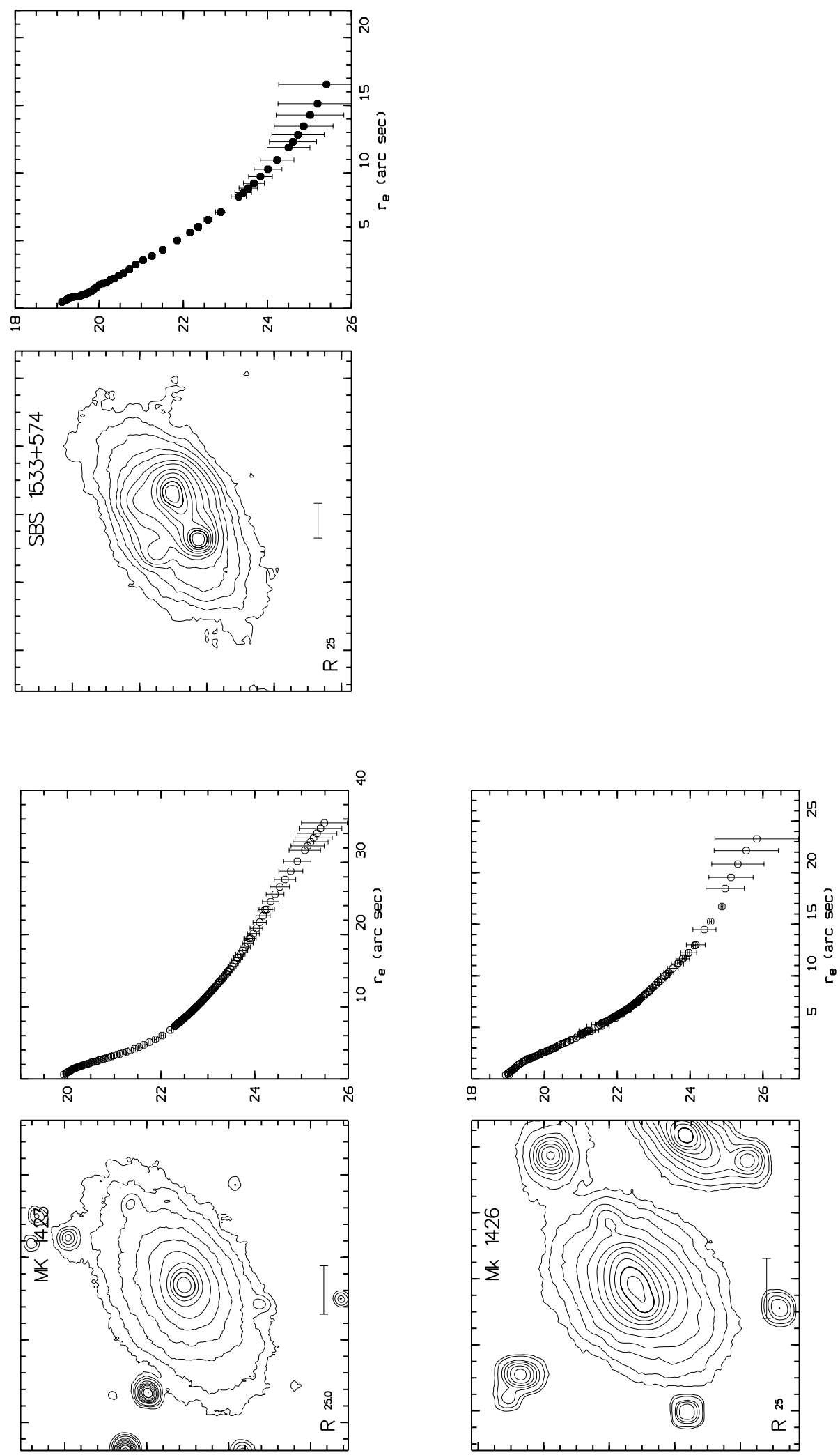

Fig. 1. continued. (To be seen in landscape) 
Table 2. Results of the photometry

\begin{tabular}{|c|c|c|c|c|c|c|c|}
\hline $\begin{array}{l}\text { Object name }[B \text { filter }] \\
\prime \prime[R \text { filter }] \\
\end{array}$ & $\begin{array}{c}m_{B} \text { (asympt) } \\
B-R \text { (asympt) }\end{array}$ & $\begin{array}{l}\mu_{\text {eff }}(B)\left[\mathrm{mag} / \operatorname{arcsec}^{2}\right] \\
\mu_{\mathrm{eff}}(R)\left[\mathrm{mag} / \operatorname{arcsec}^{2}\right]\end{array}$ & $\begin{array}{l}<\mu_{\text {eff }}>[B] \\
<\mu_{\text {eff }}>[R]\end{array}$ & $\begin{array}{l}r_{\text {eff }}(B)\left[{ }^{\prime \prime}\right] \\
r_{\text {eff }}(R)\left[^{\prime \prime}\right] \\
\end{array}$ & $\begin{array}{l}r_{0.25}(B)\left[{ }^{\prime \prime}\right] \\
r_{0.25}(R)\left[{ }^{\prime \prime}\right] \\
\end{array}$ & $\begin{array}{l}r_{24}(B)\left[{ }^{\prime \prime}\right] \\
r_{24}(R)\left[{ }^{\prime \prime}\right] \\
\end{array}$ & $\begin{array}{l}C_{21}(B) \\
C_{21}(R) \\
\end{array}$ \\
\hline \multirow{2}{*}{$\overline{\mathrm{SBS}^{\prime} 0136+328}$} & 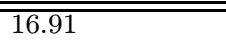 & 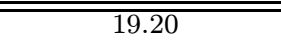 & 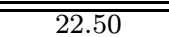 & 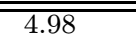 & $\overline{2.38}$ & 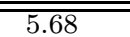 & $\overline{2.09}$ \\
\hline & 1.03 & 16.68 & 20.40 & 3.08 & 1.78 & 9.04 & 1.73 \\
\hline \multirow{2}{*}{$\underset{\prime \prime}{\text { SBS } 0940+544 \mathrm{C}}$} & 17.47 & 24.12 & 23.51 & 6.14 & 3.59 & 3.92 & 1.71 \\
\hline & 0.65 & 22.94 & 22.30 & 4.81 & 2.72 & 7.8 & 1.77 \\
\hline \multirow{2}{*}{$\underset{\prime \prime}{\mathrm{SBS}} 1006+578$} & 16.55 & 22.38 & 21.81 & 4.28 & 2.22 & 7.75 & 1.92 \\
\hline & 0.88 & 22.26 & 21.47 & 5.59 & 3.31 & 10.22 & 1.69 \\
\hline \multirow{2}{*}{$\underset{\prime \prime}{\text { SBS } 1054+504}$} & 16.19 & 22.33 & 21.37 & 4.11 & 2.01 & 7.81 & 2.04 \\
\hline & 1.04 & 21.73 & 20.67 & 4.86 & 2.31 & 12.64 & 2.11 \\
\hline \multirow{2}{*}{$\underset{\prime \prime}{\mathrm{SBS}} 1147+520$} & 17.29 & 22.27 & 21.81 & 2.88 & 1.58 & 4.85 & 1.82 \\
\hline & 1.45 & 20.90 & 20.37 & 3.01 & 1.86 & 8.17 & 1.62 \\
\hline \multirow{2}{*}{$\underset{\prime \prime}{\mathrm{SBS}} 1331+493$} & 15.03 & 18.77 & 22.63 & 12.52 & 7.50 & 15.48 & 1.67 \\
\hline & 0.61 & 18.17 & 22.04 & 12.91 & 7.73 & 20.09 & 3.09 \\
\hline \multirow{2}{*}{$\underset{\prime \prime}{\text { SBS } 1413+495}$} & 16.40 & 20.23 & 23.78 & 15.78 & 6.35 & 8.84 & 2.44 \\
\hline & 0.82 & 18.01 & 22.77 & 9.98 & 3.22 & 10.13 & 3.09 \\
\hline \multirow{2}{*}{$\underset{\prime \prime}{\text { SBS } 1428+457}$} & 16.02 & 22.07 & 21.27 & 4.48 & 2.61 & 9.44 & 1.98 \\
\hline & 1.45 & 20.75 & 19.86 & 4.56 & 2.13 & 13.36 & 2.14 \\
\hline \multirow{2}{*}{$\underset{\prime \prime}{\text { SBS } 1533+574}$} & - & - & - & - & - & & \\
\hline & $15.36\left(m_{r}\right)$ & 21.22 & 20.50 & 4.11 & 2.01 & 10.28 & 2.04 \\
\hline \multirow{2}{*}{$\underset{\prime \prime}{\operatorname{MRK} 324}$} & 14.91 & 19.63 & 22.07 & 10.40 & 3.06 & 9.57 & 3.40 \\
\hline & 0.41 & 16.56 & 20.01 & 4.92 & 2.70 & 12.91 & 1.82 \\
\hline \multirow{2}{*}{$\underset{\prime \prime}{\text { MRK } 900}$} & 14.38 & 19.23 & 22.58 & 16.19 & 5.63 & 14.31 & 2.87 \\
\hline & 1.47 & 18.41 & 21.70 & 21.75 & 8.44 & 36.34 & 2.58 \\
\hline \multirow{2}{*}{$\underset{\prime \prime}{\text { MRK } 996}$} & - & - & - & 5.74 & 2.58 & 15.74 & 2.22 \\
\hline & - & - & - & 14.94 & 5.92 & 19.66 & 2.52 \\
\hline \multirow{2}{*}{$\begin{array}{l}\text { Mk } 1131 \\
\prime \prime\end{array}$} & 15.03 & 19.76 & 23.58 & 19.77 & 9.12 & 10.61 & 2.16 \\
\hline & - & - & - & 7.34 & 3.62 & 22.63 & 2.03 \\
\hline \multirow{2}{*}{$\underset{\prime \prime}{\text { MK } 1308}$} & 14.87 & 22.68 & 21.45 & 8.17 & 2.55 & 15.06 & 3.21 \\
\hline & 1.14 & 21.06 & 20.10 & 7.44 & 3.12 & 22.51 & 2.38 \\
\hline \multirow{2}{*}{ MK 1416} & 16.99 & 22.93 & 22.07 & 4.14 & 2.19 & 6.75 & 1.89 \\
\hline & 1.29 & 18.87 & 20.94 & 4.49 & 2.00 & 9.81 & 2.24 \\
\hline \multirow{2}{*}{$\underset{\prime \prime}{\mathrm{MK}} 1418$} & 14.37 & 21.56 & 20.85 & 7.79 & 4.90 & 16.95 & 1.59 \\
\hline & 1.46 & 20.50 & 19.81 & 9.10 & 5.44 & 28.09 & 1.67 \\
\hline \multirow{2}{*}{ MK 1423} & - & - & - & - & - & & \\
\hline & $13.76\left(m_{r}\right)$ & 18.78 & 21.41 & 13.42 & 5.84 & 20.39 & 2.30 \\
\hline \multirow{2}{*}{$\underset{\prime \prime}{\mathrm{MK}} 1426$} & & - & - & - & - & & \\
\hline & $14.76\left(m_{r}\right)$ & 21.32 & 20.22 & 4.89 & 2.30 & 14.45 & 2.12 \\
\hline \multirow{2}{*}{$\underset{\prime \prime}{\operatorname{MK} 1434}$} & 17.36 & 22.01 & 20.86 & 2.00 & 1.06 & 4.79 & 1.88 \\
\hline & 1.17 & 20.91 & 19.80 & 2.10 & 1.07 & 6.90 & 1.97 \\
\hline \multirow{2}{*}{$\begin{array}{l}\text { MK } 1450 \\
\prime \prime\end{array}$} & 16.99 & 21.68 & 20.71 & 2.22 & 1.22 & 5.33 & 1.82 \\
\hline & 2.16 & 19.76 & 18.78 & 2.46 & 1.37 & 11.17 & 1.79 \\
\hline \multirow{2}{*}{$\underset{\prime \prime}{\operatorname{MK} 1480}$} & 16.56 & 21.95 & 21.11 & 3.25 & 1.64 & 6.69 & 1.97 \\
\hline & 1.03 & 21.60 & 20.65 & 4.21 & 2.13 & 10.37 & 1.98 \\
\hline \multirow{2}{*}{$\underset{\prime \prime}{\text { MK } 1481}$} & 16.78 & 23.53 & 22.83 & 6.47 & 3.70 & 8.05 & 1.75 \\
\hline & 0.79 & 22.99 & 22.24 & 7.13 & 3.95 & 10.14 & 1.81 \\
\hline MK 1499 & 16.53 & 22.43 & 21.49 & 3.90 & 1.97 & 6.82 & 1.98 \\
\hline & 1.10 & 21.41 & 21.16 & 5.55 & 2.47 & 12.40 & 2.24 \\
\hline
\end{tabular}

4 knots of star formation activity. In most cases, the knots have a different $B-R$ color as their environment. They can be classified from red to blue, this probably reflecting different states of evolution, the brightness of the reddest knots being possibly dominated by red supergiants. Usually the bluest knots are off-centered and may contribute significantly to the luminosity in the blue range.

A substantial fraction of the sample shows outer isophotes that depart conspicuously from axisymmetric shapes. As examples, SBS0940+544C and Mk 1416 which show a comet-like shape. Although it is tempting to associate these departures from axisymmetry with recent gravitational interactions of tidal type with other systems, we have found only two objects with clearly identified com- panions, namely Mk 1308 associated with a very close-by dwarf Magellanic companion (2 kpc in projected distance), and Mk 1480 whose companion Mk 1481 is at about $11 \mathrm{kpc}$ from its center in projected distance. A number of objects also show distortions in more inner regions, the most common behavior being the "boxy" nature of isophotes (that may extend to almost rectangular or lozenge shapes in extreme cases) and the rotation of the position angle of the apparent major axis of the isophotes. It has been widely claimed in the literature that boxy isophotes in spheroidal systems may be signatures of past gravitational interactions and/or current merging phenomena seen just before the complete relaxation of the system (Nieto \& Bender 1989; Hearnquist \& Quinn 1989; Jedrzejewski et al. 1987; 
Barnes \& Hearnquist 1992; Nieto et al. 1994).

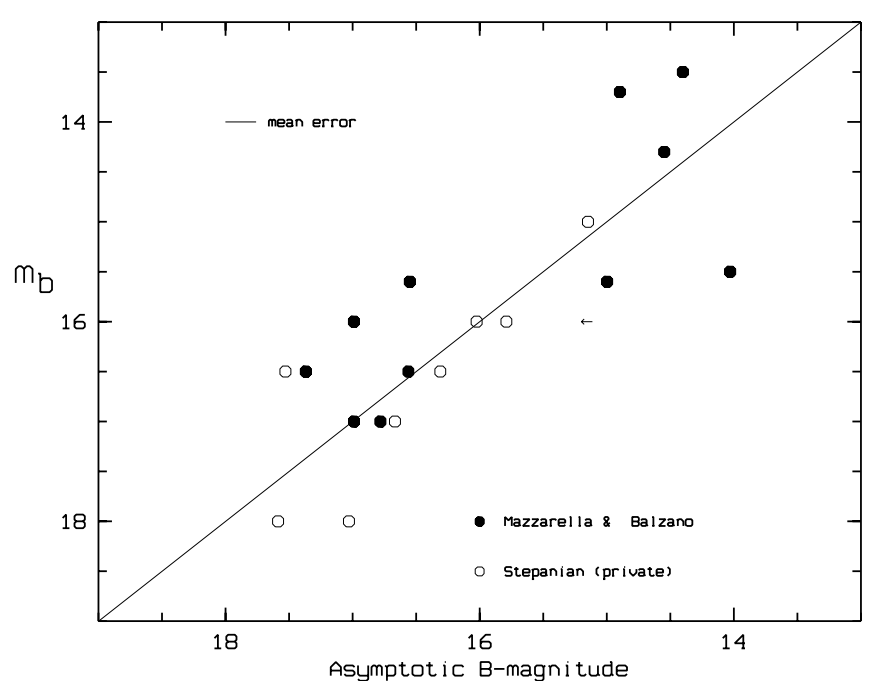

Fig. 2. Comparison between photographic apparent magnitudes from Byurakan surveys and asymptotic $B$ magnitudes in this work. The sources of photographic magnitudes are Mazzarella \& Balzano (1988) (filled hexagon) and Stepanian (private communication, open circle). The right arrows represent Mk 1131 and Mk 996 for which the photometric data are not reliable because of very poor weather conditions during the observing run

Below are listed short descriptions of the objects.

- SBS 0136+328: The most distant and most luminous object in our sample, it shows an elliptical shape with no apparent substructure. The exact nature of the brightness distribution is difficult to assess with certainty because of the limited scale $\left(1^{\prime \prime}=380 \mathrm{pc}\right)$, the most probable case is a large, dominant $r^{1 / 4}$ bulge plus exponential envelope. The photometry is uneasy due to a bright star projected near the galaxy and producing diffuse light in the background.

- SBS 0940+544C: The faintest object in the sample $\left(M_{B}=-13.9\right)$. Elongated diffuse galaxy, with an extended central region of quite uniform brightness. A Galactic star is superimposed at one extremity. Numerous faint galaxy images in the vicinity, probably distant background objects. The brightness distribution obeys a quasi-pure exponential law.

- SBS 1006+578: Outer contours are roughly rectangular in shape, with a brighter extended knot at the SW edge (there remains a little suspicion that this knot could be a background object) and a weak extension along the major axis. The central star forming region is kidney-shaped, with no apparent structure in it. A strong color gradient is present, the outer envelope being very red. The brightness distribution looks exponential, but in the red band the profile is composite with the outer envelope having a scale length different from the one observed in the central region. This anomalous morphology may result from past tidal interactions, but no neighbors have been identified so far.

- SBS 1054+504: A quite regular elliptical-like galaxy with some isophote rotation between the central part and the outer envelope and evidences for local departure from axisymmetry. The brightness distribution obeys a $r^{1 / 4}$ law, with a clear and regular color gradient, the outer envelope being redder.

- SBS 1147+520: A small galaxy with roughly regular outer isophotes. A conspicuous diffuse component appears at the NW. The central bright star forming region is elongated and kidney-like. The brightness distribution shows a trend to obey a linear relationship between $\mu$ and $r^{1 / 4}$ but the coefficient is not typical of normal spheroidal systems (see discussion in Sect. 4.1.1). A weak color gradient is present.

- SBS 1331+493: Classified as a compact dwarf because of the luminous blue knot located E (Movsesyan et al. 1996): it is a giant starforming region with strong $\mathrm{H} \alpha$ and [OIII] lines and a very weak continuum. The galaxy shows an amorphous shape and the brightness distribution does not obey standard laws. We have constructed a $B-R$ map that shows complex structures in the central region with several faint blue enhancements.

- SBS 1413+495: Elliptic shape with very regular isophotes. Unfortunately the presence of a Galactic star very close-by and the poor seeing conditions do not allow a more accurate description of this object. Bluest galaxy of our sample $(B-R=0.22$ in asymptotic value), but this extreme color is due to an extremely blue envelope, the core color being normal.

- SBS 1428+457: The overall shape is a deformed lozenge, whose central part is a very high surface brightness star forming region. The overall color is not very blue, and there is no appreciable color gradient. The brightness distribution roughly follows an exponential law with a central excess of light. A lot of structures are present across the inner regions, four plumes each containing $2-3$ resolved knots protrude out of the central bright core. A compact object of neutral color, apparently a superimposed Galactic star, is conspicuous near the S end.

- SBS 1533+574: Only observed in $R$ band. The outer isophotes are elliptic and quite regular. The central region is dominated by 2 bright components, one compact and stellar-like, the other more diffuse and elongated towards the former. Both are off-centered with respect to the outer isophotes. Two secondary components at north form a trapezoidal structure with the main cores. This object may perhaps be linked to or classified as a double nucleus galaxy. The brightness 
distribution obeys an exponential law with evidence for an outer envelope having a shallower gradient.

- Mk 324: A quite regular elliptical double-nucleated galaxy (Barbieri et al. 1979; Korovyakovskii et al. 1981). The two "nuclei" differ in angular extent and compactness. Both are off-centered with respect to the outer contours. The brightness distribution follows an $r^{1 / 4}$ law. There is a strong color gradient, the envelope being bluer than the inner region. The photometry presented here has uncertain calibration because of poor atmospheric conditions during the observations.

- Mk 900: Elliptical shape with isophotes showing rotation and boxiness. The central part of the galaxy is elongated and strongly distorted, especially on the $R$ frame. The brightness distribution is consistent with an $r^{1 / 4}$ law in the central region, but there is evidence for an extended envelope of different dynamical behaviour.

- Mk 996: Typical elliptical-like galaxy, with rotating isophotal major axis and surface brightness light profiles described by a pure $r^{1 / 4}$ law. Qualitative photometry and morphology could only be done on this galaxy because of the very poor photometric conditions during the observation.

- Mk 1131: Elliptic shape with off-centered central core. The isophotes are regular ellipses, the brightness distribution is peculiar with a very shallow outer envelope surrounding the steeper central population. The photometric calibration is quite uncertain because of poor weather conditions.

- Mk 1308 (= UM 465): Brightest component of a pair, a faint irregular companion (confirmed as physical neighbour by $6 \mathrm{~m}$ telescope spectroscopy) is located at $32^{\prime \prime}(2 \mathrm{kpc})$. The envelope of Mk 1308 is roughly circular in shape but the isophotes exhibit departures from symmetry. The brightness distribution follows an exponential law. Salzer et al. (1989) describe this object as a "nearby dwarf elliptical" with a "nuclear starburst" but we shall notice that the brightness distribution in this case rather corresponds to that observed in non-active dwarf spheroidal objects (see e.g. Ichikawa et al. 1986) and not to that observed in giant ellipticals.

- Mk 1416: Comet-like galaxy with elongated boxy isophotes. The brightest region exhibits a double component structure: one component approximately located at the center has a red color while the second component is very blue. An extended "transversal" structure is visible in the half of the galaxy opposite from the blue compact component. The brightness distribution could be roughly consistent with a superimposition of two exponential laws. A faint red envelope is detectable near the surface brightness limit of the images.

- MK 1418: A nearby dwarf galaxy. The external isophotes are oval. The central bright region is very extended, with several protruding blobs out of a distorted central component surrounding a small bright nucleus. The brightness distribution follows an $r^{1 / 4}$.

- Mk 1423: Only observed in $R$ band. Oval and regular in shape with an asymmetric bright core. The brightness distribution is consistent with a disk plus bulge population superimposition.

- Mk 1426: Only observed in $R$ band. Another, fainter galaxy at $48^{\prime \prime}$ but we have no information about its velocity. The outer shape is elliptic. The central bright starburst region is a double component embedded in an elongated envelope. The brightest component is centered with respect to outer isophotes. The brightness distribution follows an $r^{1 / 4}$ law.

- Mk 1434: Distant dwarf object with lozenge-like outer contours and a jet-like curved extension at one end. The central bright component is ovoid in shape. There is no significant color gradient. The brightness distribution obeys an $r^{1 / 4}$ law.

- Mk 1450: A dwarf compact object with moderately deformed circular isophotes and a unique central star forming component. The brightness distribution obeys an $r^{1 / 4}$ law. A strong color gradient is present, the envelope being very red. Note that the central component is also quite red for a starburst object.

- Mk 1480: In physical pair with Mk 1481 (see below). This galaxy is quite regular with a faint extension opposite to the direction of the companion and boxy isophotes. The bright core is centrally located. The brightness distribution obeys an $r^{1 / 4}$ law, with a strong and regular color gradient, the envelope being much redder than the center.

- Mk 1481: Small irregular object of Magellanic aspect with complicated inner structure of several bright spots embedded in a common envelope. The brightness distribution follows an exponential law with a shallow gradient. The outer envelope is redder than the central parts.

- Mk 1499: Boxy (almost rectangular) inner body embedded in an elliptical extended envelope in which there are some hints of very faint shells corresponding to the small sides of the inner "box". The inner body itself contains a bright elongated very blue central structure with two components, the brightest being off-centered with respect to the outer contours. This structure is reminiscent of "double nuclei" objects. The brightness distribution is neither consistent with an exponential nor with an $r^{1 / 4}$ law.

\section{Discussion}

\subsection{The surface brightness distributions}

On the atlas of profiles, (Fig. 1), a classical flat (disk) distribution of light, following the well-known exponential law, translates into a linear relation. Alternatively, 
a plot of the surface brightness versus the 0.25 power of the equivalent radius allows to recognize the objects having a light distribution of "spheroidal galaxy" type. One would expect the occurrence of "mixed" profiles indicating the probable presence of two dynamically different populations, the disk-like one and the spheroidal one, making such objects low luminosity equivalents of normal giant flattened galaxies.

The preliminary study of the brightness profiles allows us to sort out:

-10 objects with a $r^{1 / 4}$ law profile,

- 7 objects with exponential profiles and sometimes a brightness excess limited to the very central area.

- 6 objects with profiles departing conspicuously from the standard laws, among these, 4 can be interpreted by a superposition of an exponential brightness distribution with a spheroidal one.

In the following we discuss some characteristics of the derived surface brightness profiles.

\subsubsection{Objects with an $r^{1 / 4}$ law}

Figure 3 shows, in $\left(\mu,\left(r_{\mathrm{e}} / r_{\text {eff }}\right)^{1 / 4}\right)$ coordinates, the observed brightness distribution of the 10 objects exhibiting a linear $r^{1 / 4}$ law across a substantial range of equivalent radius. We assume that the surface brightness profile follows the empirical law as given by De Vaucouleurs (1948, 1953)

$\log \frac{I}{I_{\text {eff }}}=-3.3307\left[\left(\frac{r}{r_{\text {eff }}}\right)^{1 / 4}-1\right]$

where $I$ is the intensity at a given $r$ and $I_{\text {eff }}$ is the intensity at the effective radius. This relation is more convenient to use in terms of surface brightness $\mu$

$\mu-\mu_{\mathrm{eff}}=8.327\left[\left(\frac{r}{r_{\mathrm{eff}}}\right)^{1 / 4}-1\right]$.

Genuinely fitting a linear relation on the ( $\mu$ versus $\left.\left(r_{\mathrm{e}} / r_{\mathrm{eff}}\right)^{1 / 4}\right)$ plot of the surface brightness in the range of $r_{\mathrm{e}}$ not affected by seeing effects, we get the slope and the deviation of the profile from the pure $r^{1 / 4}$ law. It appears that for some galaxies (Mk 1450, Mk 1480 and SBS $1147+520)$ the slope is different in the two bands. Note that the 8.327 slope is intrinsic to the $r^{1 / 4}$ law, as written in terms of normalized coordinates $\left(\mu-\mu_{\mathrm{eff}}\right.$, $\left.\left(r_{\mathrm{e}} / r_{\mathrm{eff}}\right)^{1 / 4}\right)$. A value of the slope differing significantly from the canonical 8.327 usually implies that the adopted $r_{\text {eff }}$ is not correct. Since the determination of $r_{\text {eff }}$ is made through an extrapolation process (in order to derive the asymptotic magnitude from the cumulated magnitude growth curve) the errors are quite difficult to control, especially when the frames are insufficiently deep. However, another effect is also responsible for the deviation from a pure $r^{1 / 4}$ law: if the galaxy houses a population dynamically different from an isothermal spheroid (King 1966) that contaminates the light of the latter, departure from the $r^{1 / 4}$ law will be quickly visible. This is the obvious case for extended envelopes that show up as excess light above the $r^{1 / 4}$ (e.g. SBS0136+328, Mk 324 and Mk 1426). It should be underlined that these outer envelopes differ in color from that of more central regions, suggesting a different stellar content. Also, if the $\left[\mu,\left(r_{\mathrm{e}} / r_{\text {eff }}\right)^{1 / 4}\right]$ relationship is linear but with a slope different from 8.327, on frames sufficiently deep such as to rule out significant errors on $r_{\text {eff }}$, it could be suggested that a non-spherical population is quite continuously superimposed to the spherical one, (but with a moderate contribution to the total light) so that the scale length is changed (as could be the case for Mk 324).

\subsubsection{Exponential profiles}

In most cases, the exponential law (de Vaucouleurs 1959) applies right after the seeing limit $\left(r_{\mathrm{e}}=0.5\right.$ to $\left.3^{\prime \prime}\right)$.

$I(r)=I_{0} \exp \left(-\frac{r}{r_{0}}\right)$

or, in terms of surface brightness:

$\mu=\mu_{0}+1.0857\left(\frac{r}{r_{0}}\right)$

where $1 / r_{0}$ is the scaling factor in $\mathrm{kpc}^{-1}$ and $\mu_{0}$ is the central value of the surface brightness. In other cases, there is a significant contribution of a small brighter central component. See also the case of SBS $1006+578$ in Sect. 3.5. Table 4 gives the resulting numerical values of the linear regression.

\subsubsection{Mixed law profiles}

4 galaxies show a profile reminiscent of the classical superimposition of a central bulge component with an underlying exponential law extending to the outer parts where this latter becomes dominant. The exponential is easily interpreted as showing up the presence of a disk. Only 2 cases show a slight systematic blueing outwards. (SBS $1413+495$ and SBS 0940+544C)

\subsection{Integral relationships}

Table 4 lists the integrated parameters on the observed galaxies together with metric observables deduced from the photometry. The distance dependent quantities have been derived using $H_{0}=80 \mathrm{~km} \mathrm{~s}^{-1} \mathrm{Mpc}^{-1}$, the redshifts of Table 2 corrected from Galactic rotation (using $\left.V_{\text {cor }}=V_{\odot}+300 \times \cos b\right)$ and from the Virgo-centric infall model of Aaronson et al. (1981) as reformulated by Bottinelli et al. (1986) using the recent Virgo distance 
Table 3. Exponential profiles

\begin{tabular}{rcccccc}
\hline \hline name & $r_{\mathrm{e}}(R)[\mathrm{pc}]$ & $r_{\mathrm{e}}(B)[\mathrm{pc}]$ & $\frac{1}{r_{0}}(R)\left[\mathrm{kpc}^{-1}\right]$ & $\frac{1}{r_{0}}(B)\left[\mathrm{kpc}^{-1}\right]$ & $\mu_{0}(R)$ & $\mu_{0}(B)$ \\
\hline sbs0940 & 308 & 525 & 1.12 & 1.11 & 21.78 & 22.39 \\
mrk1308 & 407 & 317 & 1.31 & 1.20 & 19.60 & 21.09 \\
sbs1006 & 430 & 338 & 0.40 & 0.51 & 20.07 & 20.64 \\
mrk1416 & 478 & 518 & 1.19 & 1.04 & 20.11 & 20.61 \\
sbs1428 & 555 & 597 & 1.03 & 0.98 & 18.9 & 20.37 \\
sbs1533 & 684 & - & 0.97 & - & 18.38 & - \\
mrk1423 & 779 & - & 0.65 & - & 15.06 & - \\
& & & & & & \\
\end{tabular}

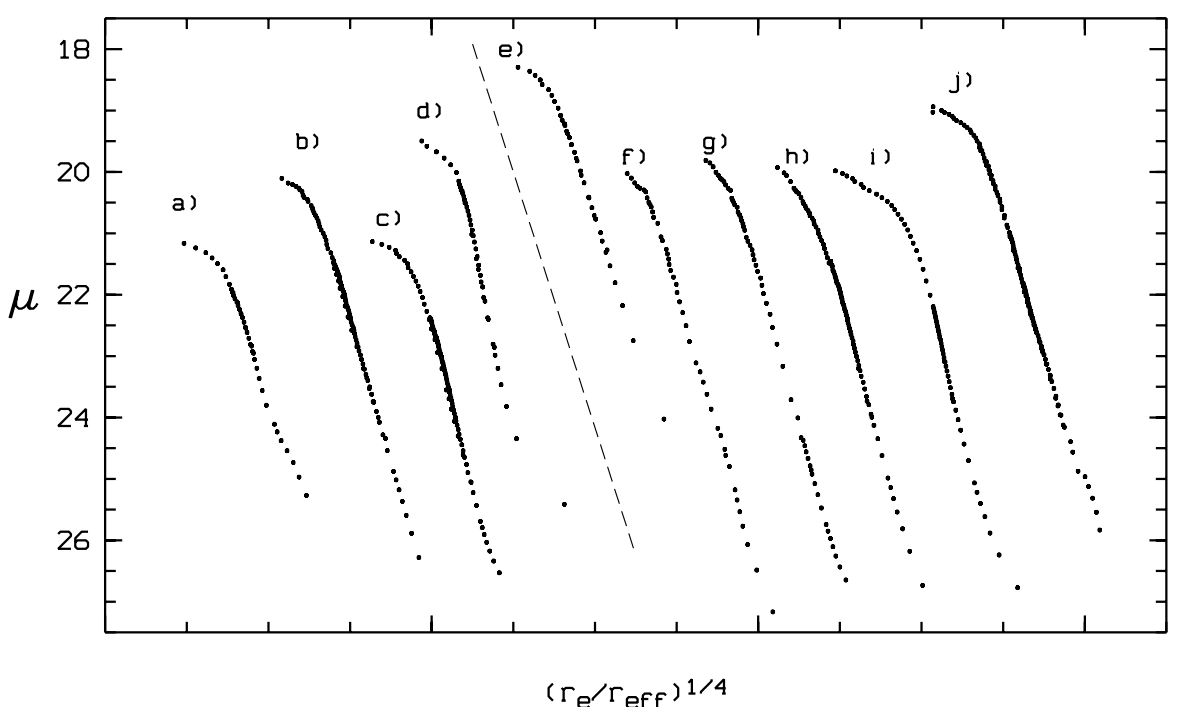

Fig. 3. Reduced surface brightness distributions of the 10 galaxies obeying an $r^{1 / 4}$ law. The abscissa is the quantity $\left(r_{\mathrm{e}} / r^{\mathrm{eff}}\right)^{1 / 4}$, the ordinate is the surface brightness in magnitudes per square arc second. The tick marks in abscissa represent a 0.5 interval. The dashed line has a slope of 8.327 and represents the "pure" de Vaucouleurs' law. The various curves, translated from each other to ensure legibility, are for: a) SBS $0136+328$, b) SBS $1054+504$, c) SBS $1147+520$, d) Mk 324 , e) Mk 996, f) Mk 1434 , g) Mk 1450, h) Mk 1480, i) Mk 1418, j) Mk 1426

based on Cepheid photometry by Freedman et al. (1994).

Table 4 is arranged as follows:

Column 1: Name of the galaxy

Column 2: Distance in Mpc.

Column 3: Absolute $B$ magnitude from our asymptotic magnitude.

Column 4: Effective equivalent radius in pc, in $B$ and $R$ colors.

Column 5: Isophotal equivalent radius at $\mu=24$ in pc, in $B$ and $R$ colors.

\subsubsection{Color distributions}

Figure 4 displays the histogram of the asymptotic $B-R$ colors.
The asymptotic $B-R$ color ranges from 0.22 to 2.16 . The mean color of the sample is 1.09 (with no correction for reddening due to Galactic or internal absorption) which places the sample among the blue galaxy population. Fukugita et al. (1995) have recently revised the integrated colors of various galaxy types and show that normal field galaxies of elliptical types have $B-R=1.6$ while the bluest spirals of type Scd have $B-R=1.0$ (from synthetic color computation, perhaps a little redder from observations). 2 objects in our sample are very blue with $B-R \leq 0.5$ : SBS $1413+495(B-R=0.22)$ and Mk $324(B-R=0.44)$. We note that the galaxies whose brightness distribution obey an $r^{1 / 4}$ law follow the same distribution of colors as that of the whole sample. 
Table 4. Absolute magnitudes and linear radii

\begin{tabular}{|c|c|c|c|c|}
\hline$\overline{\overline{\text { Object name }}}$ & $\overline{\bar{d}[\mathrm{Mpc}]}$ & $\overline{\bar{M} M_{B}}$ & $\begin{array}{l}r_{\text {eff }}[\mathrm{pc}](B) \\
r_{\text {eff }}[\mathrm{pc}](R)\end{array}$ & $\begin{array}{l}r_{24}[\mathrm{pc}](B) \\
r_{24}[\mathrm{pc}](R)\end{array}$ \\
\hline \multirow[t]{2}{*}{ SBS 0136} & 84.5 & -17.72 & 1910 & 2181 \\
\hline & & & 1184 & 3472 \\
\hline \multirow{2}{*}{ SBS 0940} & 23.0 & -14.34 & 643 & 577 \\
\hline & & & 503 & 638 \\
\hline \multirow[t]{2}{*}{ SBS 1006} & 18.6 & -14.80 & 362 & 655 \\
\hline & & & 473 & 864 \\
\hline \multirow{2}{*}{ SBS 1054} & 18.6 & -15.16 & 348 & 661 \\
\hline & & & 411 & 1070 \\
\hline \multirow{2}{*}{ SBS 1147} & 15.0 & -13.60 & 197 & 332 \\
\hline & & & 205 & 559 \\
\hline \multirow{2}{*}{ SBS 1331} & 10.8 & -15.14 & 616 & 761 \\
\hline & & & 635 & 988 \\
\hline \multirow[t]{2}{*}{ SBS 1413} & 11.1 & -13.83 & 803 & 505 \\
\hline & & & 505 & 512 \\
\hline \multirow{2}{*}{ SBS 1428} & 32.1 & -16.51 & 653 & 1376 \\
\hline & & & 665 & 1948 \\
\hline \multirow{2}{*}{ SBS 1533} & 44.2 & -- & -- & -- \\
\hline & & & 825 & 2065 \\
\hline \multirow[t]{2}{*}{ Mk 324} & 23.9 & -16.98 & 1131 & 1041 \\
\hline & & & 536 & 1405 \\
\hline \multirow[t]{2}{*}{ Mk 900} & 17.5 & -16.83 & 1286 & 1137 \\
\hline & & & 1729 & 2888 \\
\hline \multirow[t]{2}{*}{ Mk 996} & 21.4 & -- & 558 & 1532 \\
\hline & & & 1454 & 1913 \\
\hline \multirow[t]{2}{*}{ Mk 1131} & 27.3 & -17.24 & 2453 & 1316 \\
\hline & & & 911 & 2808 \\
\hline \multirow[t]{2}{*}{ Mk 1308} & 13.6 & -15.80 & 505 & 931 \\
\hline & & & 460 & 1392 \\
\hline \multirow[t]{2}{*}{ Mk 1416} & 28.7 & -15.31 & 541 & 881 \\
\hline & & & 586 & 1281 \\
\hline \multirow{2}{*}{ Mk 1418} & 6.3 & -14.62 & 223 & 484 \\
\hline & & & 260 & 803 \\
\hline \multirow[t]{2}{*}{ Mk 1423} & 15.5 & -- & -- & -- \\
\hline & & -17.17 & 948 & 1441 \\
\hline \multirow[t]{2}{*}{ Mk 1426} & 25.2 & -- & -- & -- \\
\hline & & -17.23 & 561 & 1658 \\
\hline \multirow[t]{2}{*}{ Mk 1434} & 27.6 & -14.84 & 251 & 601 \\
\hline & & & 264 & 866 \\
\hline \multirow[t]{2}{*}{ Mk 1450} & 12.6 & -13.52 & 127 & 306 \\
\hline & & & 141 & 641 \\
\hline \multirow[t]{2}{*}{ Mk 1480} & 24.3 & -15.36 & 358 & 738 \\
\hline & & & 465 & 1144 \\
\hline \multirow[t]{2}{*}{ Mk 1481} & 25.1 & -15.21 & 737 & 917 \\
\hline & & & 812 & 1154 \\
\hline \multirow[t]{2}{*}{ Mk 1499} & 36.7 & -16.29 & 650 & 1138 \\
\hline & & & 926 & 2068 \\
\hline
\end{tabular}

\subsubsection{Radius-luminosity relations}

Figure 5a shows relations between the logarithm of the effective radius and the absolute magnitude in $B$, uncorrected for Galactic and internal absorption. The two quantities are correlated (correlation coefficient of 0.82 in $B$ and 0.66 in $R$ ) as expected, with a slope of $-3.65 \pm 1.08$ in $B$ and $-5.14 \pm 2.06$ in $R$.

In $B$, there is a clear deviation from the expected slope of -5 implied by a dependence of the luminosity on the square of the radius. It should be remembered that Heidmann (1969) found a dependence of the luminosity on $r^{k}$ with $k=1.9$ for giant ellipticals and $k=2.8$ for giant spirals. However Fraser (1977) found $k=2.1$ for Virgo spirals). The tendency in our sample is towards an excess
$B$ luminosity for the less luminous objects, for a given effective radius. Although the dispersion in both colors may be explained partly by the extrapolation errors in the determination of $r_{\text {eff }}$ (see Sect. 4.1.2) and the errors on the distance moduli, the trend would rather be due to the selection bias of the sample. The most extreme blue compact dwarfs are selected in the Byurakan surveys because their central surface brightness in the blue part of the spectrum, reinforced by the starburst, is intrinsically high. The use of $r_{\text {eff }}$ favours the weight of the central regions where the burst dominates the light (see Table 4), and this bias is stronger on the intrinsically faint objects.

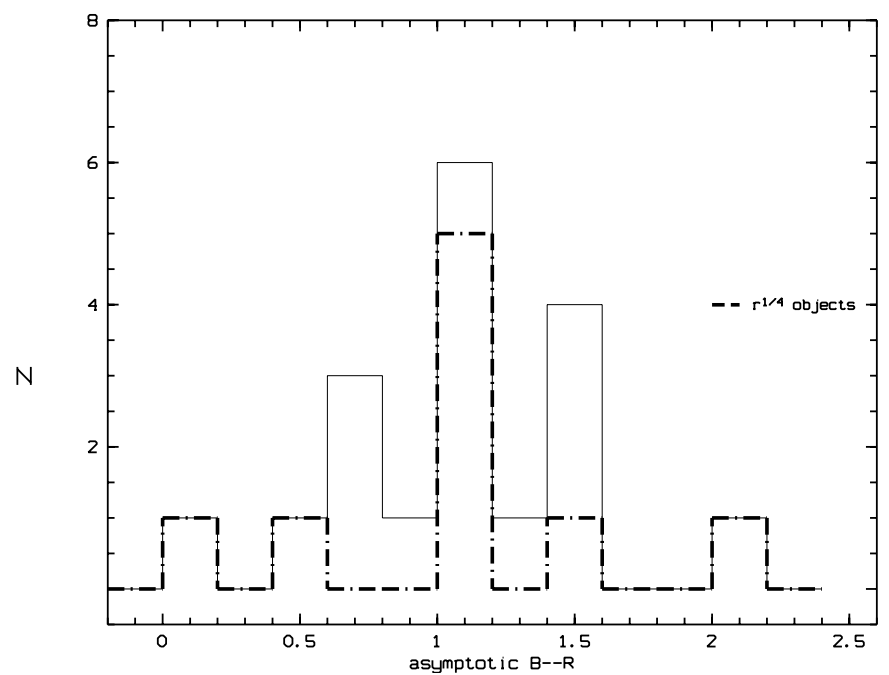

Fig. 4. Distribution of asymptotic $B-R$ colors

In $R$ band, there is a large dispersion of the representative points, but, as expected, the "blue" selection bias as explained above is much less apparent.

Figure 5b shows the same relationship using the isophotal radius $r_{24}$. This radius is systematically larger than $r_{\text {eff }}$, and therefore integrates light largely emitted by the underlying evolved population, hence reducing the weight of the starburst component. The dispersions are much reduced, the slopes are $-6.06 \pm 1.25$ in $B$ and $-5.32 \pm 0.70$ in $R$ with respective correlation coefficients of 0.90 and 0.96 . The slope in $B$ is significantly different from -5 , but in the opposite sense as observed in the $\left(M_{B}, \log \left(r_{\text {eff }}\right)\right.$ plot. Most of the discrepancy comes from Mk 324 and Mk 1131 which both exhibit an extended envelope that contributes significantly to the light beyond $r_{24}$. Excluding these galaxies, we find a slope of $-5.39 \pm 0.71$ (correlation coefficient: 0.97$)$ for the $\left(M_{B}, r_{24}\right)$ diagram, and hence an almost identical behaviour in $B$ and $R$, and consistency with a luminosity proportional to the square of the isophotal radius.

Huchra (1977) found that the luminosity of Markaryan galaxies, irrespective of their morphological type, closely 

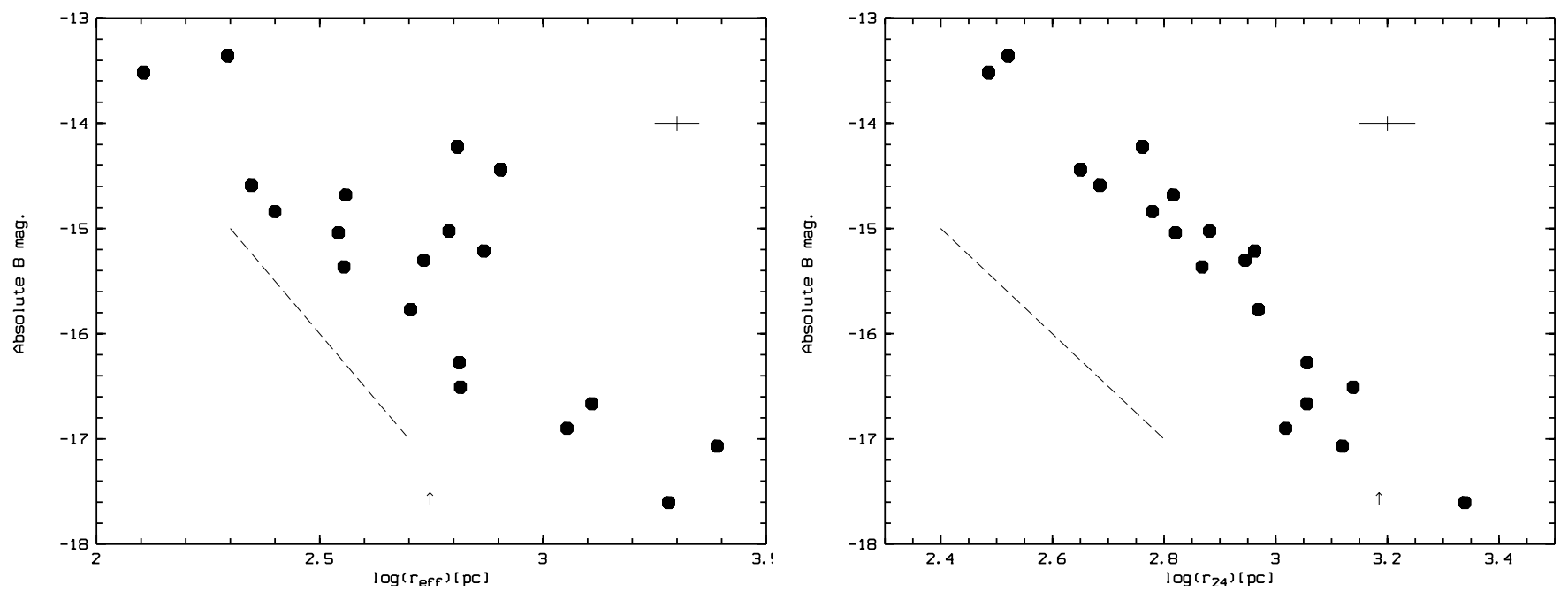

Fig. 5. Absolute magnitude-radius relations: a) at $r=r_{\text {eff }}$, (effective) in $B$ band, b) at $r=r_{24}$, (isophotal at $\mu=24$ mag arcsec ${ }^{2}$ ) in $B$ band

follows a $r^{2}$ dependence ( $r$ being an estimate of the isophotal radius), "indicating approximate constancy of mean surface brightness over the absolute magnitude interval studied". However, there is only a small number of dwarf Markaryan $\left(M_{B} \geq-16.5\right)$ in Huchra's study, and the selection effects towards compact, i.e. high surface brightness objects, in our sample are certainly different from those present in an all-type Markaryan galaxy sample.

\subsubsection{Compactness}

Compact galaxies were defined by Zwicky $(1967,1971)$ as being galaxies for which the central surface brightness is brighter than $20 \mathrm{mag} \operatorname{arcsec}^{-2}$ in $B$. But this definition applies, at least partly, to almost all galaxies having giant star-forming regions and/or a bright nuclear component. It is usually assumed that a dwarf "compact" galaxy associates two basic features: a short scale length of the brightness distribution and a high "average" surface brightness, this latter being the observable translation of a higher than normal stellar luminosity density.

Figure 6 displays the histogram of the mean surface brightness inside the effective radius measured in $B$ band. The average surface brightness of the night sky is around $\mu_{B}=22 \mathrm{mag} \operatorname{arcsec}^{-2}$. The median value, (uncorrected for Galactic foreground extinction) observed for our sample is $21.7 \mathrm{mag} \operatorname{arcsec}^{-2}$ and several objects have especially bright values (namely Mk 996, Mk 1418, Mk 1434 and Mk 1450). Compared to a sample of DDO dwarf galaxies (de Vaucouleurs et al. 1981) that exhibit low or moderate star formation rates in the average, and is largely dominated by disk late-type systems, the Byurakan dwarf blue compacts are brighter in $\left\langle\mu_{\mathrm{eff}}\right\rangle$ $(B)$ by some two magnitudes. From Ichakawa et al. (1986), we get that the average of $\left\langle\mu_{\text {eff }}>(B)\right.$ in 69 dwarf ellipticals of the Virgo Cluster is $23.5 \mathrm{mag} \operatorname{arcsec}^{-2}$. The
Byurakan BCDGs among which we find a high proportion of dwarf elliptical galaxy analogs (but with $r^{1 / 4}$ laws while dE's of Virgo are claimed to have exponential brightness distributions: Ichikawa et al. 1986) are again some two magnitudes brighter.

Figure 7 displays the histogram of the concentration index $C_{21}(B)$ as defined in Sect. 3.4.1. This index averages from 1.6 to 1.9 for diffuse late-type spirals, and reaches about 2.5 to 2.8 for normal ellipticals (Fraser 1972, 1977; de Vaucouleurs \& Aguero 1973) while diffuse dwarf magellanic have $C_{21}(B) \sim 1.5$ (Ables 1971). The present sample peaks at 1.9 and one may notice three anomalously "concentrated" objects (namely Mk 324, Mk 900 and Mk 1308).

Finally, Fig. 7 shows the histogram of the effective radii in pc measured in $B$ band. The bulk of our galaxies have indeed small effective radii with 9 objects below $500 \mathrm{pc}$.

\section{Conclusion and summary}

We report the results of a surface photometry study of a sample of 23 Blue Compact Dwarf Galaxies, selected from the two surveys of Byurakan. An atlas of isophotal maps and brightness distribution profiles has been produced.

From these profiles, we classify the objects in three groups:

1) 7 objects whose brightness distributions are described by an exponential law, supposed to be characteristic of disk-like (flattened) galaxies. These objects seem to be closely related to classical Magellanic irregulars. We could not draw any conclusion on their flattening without 
Special thanks go to Jivan Stepanian who kindly communicated finding charts, accurate coordinates, redshifts and provisional magnitudes for many unpublished objects in the SBS.

\section{References}

Aaronson M., Huchra J., Mould J., Schechter P.L., Tully R.B., 1982, ApJ 258, 64

Aaronson M., 1986, in Star-forming Galaxies and related Problems, Kunth D., Thuan T.X. and Tran Thanh Van J. (eds.). Editions Frontières, p. 125

Ables H.D., 1971, U.S. Naval Obs. Publ. 20, 1

Barbieri C., Bonoli C., Rafanelli P., 1979, A\&AS 37, 541

Barnes J.E., Hearnquist L., 1992, ARA\&A 30, 705

Bottinelli L., Gouguenheim L., Paturel G., Teerikorpi, P., 1986, A\&A 156, 157

Bothun G.D., Halpern J.P., Lonsdale C.J., Impey C., Schmitz M., 1989, ApJS 70, 271

Burnstein D. Heiles C., 1982, AJ 87, 1165

Chevalier C., Ilovaisky S.A., 1991, A\&A 90, 225

Davidson K., Kinman T.D., 1985, ApJS 58, 321

Davidson K., Kinman T.D., Friedman S.D., 1989, AJ 97, 1591

Dufour R.J., Hester J.J., 1990, ApJ 350, 149

Fraser C.W., 1972, The Observatory 92, 51

Fraser C.W., 1977, A\&AS 29, 161

Freedman W., et al., 1994, Nat 371, 757

Fukugita M., Shimasatu K., Ichikawa T., 1995, PASP 107, 945

Gilliland R., Duncan D.K., Brown T.M., et al., 1991, AJ 101, 541

Hearnquist L., Quinn P.J., 1989, ApJ 342, 1

Heidmann J., 1969, Astrophys. Lett. 3, 19

Hua C.T., Grundseth B., Nguyen-Trong T., 1987, Ap. Lett. Comm. 25, 187

Huchra J., 1977, ApJS 35, 171

Hunter D.A., Thronson H.A., 1995, ApJ 452, 238

Ichikawa S.-I., Wakamatsu K.-I., Okamura S., 1986, ApJS 60, 475

Jedrzejewski R.I., Davies R.L., Illingworth G.D., 1987, AJ 94, 1508

King I.R., 1966, AJ 71, 64

Korovyakovskii Y.P., Petrosian A.R., Saakian K.A., Khachikian E.G., 1981, Afz 17, 231

Kunth D., Sargent W.L.W., 1972, ApJ 300, 496

Kunth D., Maurogordato S., Vigroux L., 1988, A\&A 204, 10
Landolt A.U., 1983, AJ 88, 439

Landolt A.U., 1992, AJ 104, 340

Lemaitre G., Picat J.P., Comte G., et al., 1996 (in preparation)

Loose H., Thuan T.X., 1983, Star-forming Galaxies and related Objects, Kunth D., Thuan T.X. and Tran Thanh Van J. (eds.). Editions Frontières, p. 73

Loose H., Thuan T.X., 1985, ApJ 299, 881

Markaryan B.E., 1967, Afz 3, 55

Markaryan B.E., Stepanyan D.A., 1983, Afz 19, 631

Mazzarella J.M., Balzano V.A., 1986, ApJS 62, 751

Nieto J.-L., Bender R., 1989, A\&A 215, 266

Nieto J.-L., Poulain P., Davoust E., 1994, A\&A 283, 1

Odewahn S.C., Bryja C., Humphreys R.M., 1992, PASP 104, 553

Papaderos P., Loose H.-H., Fricke K.J., Thuan T.X., 1996 (to appear in A\&A)

Petrosian A.R., Boulesteix J., Comte G., Kunth D., Le Coarer E., 1996, A\&A (submitted)

Savage B.D., Mathis J.S., 1979, ARA\&A 17, 73

Saglia, et al., 1996 (in preparation)

Salzer J.J., MacAlpine G.M., Boroson T.A., 1989, ApJS 70, 447

Sargent W.L.W., Searle L., 1970, ApJ 162, L155

Searle L., Sargent W.L.W., 1972, ApJ 173, 25

Schild R.E., 1983, PASP 95, 1021

Schild R.E., 1985, PASP 97, 824

Searle L., Sargent W.L.W., 1972, ApJ 173, 25

Stepanyan D.A., 1991 (private communication)

Thuan T.X., Martin G.E., 1981, ApJ 247, 823

Thuan T.X., 1983, ApJ 268, 667

de Vaucouleurs G., 1948, Ann. Astrophys. 11, 247

de Vaucouleurs G., 1953, MNRAS 113, 134

de Vaucouleurs G., 1959, in Handbuch der Physik 53, 319. Springer Verlag, Berlin

de Vaucouleurs G., 1962, IAU Symposium 15, Problems of Extragalactic Research, Mc Vittie G.C. (ed.), p. 3

de Vaucouleurs G., Aguero E., 1973, PASP 85, 150

de Vaucouleurs G., de Vaucouleurs A., Buta R., 1981, AJ 86, 1429

Zwicky F., 1967, Advances in A\&A 5, 267. Academic Press, New-York

Zwicky F., 1971, Catalogue of selected Compact Galaxies and of Post-Eruptive Galaxies 